\title{
Rapid Analysis of Alcohol Content During the Green Jujube Wine Fermentation by FT-NIR
}

\author{
Guifeng $\mathrm{Li}^{1}{ }^{\text {a }}, \mathrm{Ni} \mathrm{Yan}^{1}, \mathrm{Lu}$ Yuan${ }^{1}$, Jianhu $\mathrm{Wu}^{1}$, Junjie $\mathrm{Du}^{1}$, Yu’e Gao ${ }^{1}$, Yankun Peng ${ }^{2}$ \\ ${ }^{1}$ Department of Food Science, Shanxi Normal University, Linfen 041000 \\ ${ }^{2}$ China College of Engineering, China Agricultural University, Beijing 100083
}

\begin{abstract}
The near-infrared (NIR) spectroscopy combined with partial least square regression (PLS) were applied for the prediction of the alcohol content of jujube wine. The NIR spectroscopy was used to collect the spectral data of the jujube wine samples during fermentation and the data were used to establish the quantitative model of alcohol content to achieve rapid on-line detection. The NIR spectroscopy in the range of 950 to $1650 \mathrm{~nm}$ from jujube wine were collected and pre-treated by MSC (Multiplicative Scatter Correction) and FD (First Derivative). The alcohol content was measured with alcohol meter. Spectral wavelength selection and latent variables were optimized for the lowest root mean square errors. The results show that the FD - PLS model, which yielded $\mathrm{R}^{2}$ of 0.9246 and RMSEC of 0.6572 , is superior to the MSCPLS model. Results confirmed that NIR spectroscopy is a promising technique for routine assessment of alcohol content of jujube wine and is a viable and advantageous alternative to the chemical procedures involving laborious extractions. The feasibility of the method was thus verified.
\end{abstract}

\section{Introduction}

Jujube, is widely cultivated throughout our country, which is rich in protein, minerals and vitamins. It is a health food that integrates the functions of medicine, food and nutrition. However, due to the high output of jujube in China, there are serious unsalable products, decreased benefits and serious losses of jujube farmers, which greatly affected the sustainable development of jujube industry. In order to solve the problems of the development of jujube industry and change the current situation of the sluggish jujube industry, it is urgent to develop new jujube products and solve the production technology.

In recent years, with the development of economy and the improvement of people's quality of life, the demand for the nutritional value and health benefits of food have become higher and higher. The turning point of wine industry has quietly emerged. Fruit wine, with its high nutritional value, strong health benefits, as well as unique colour and fragrance, has won the trust and love of consumers. Immature jujube is rich in protein, active polysaccharide, various minerals and vitamins, and contains health-care ingredients such as polyphenols, flavonoids, triterpenoids [1]. It's contents of vitamins, flavonoids and triterpenoids are much higher than that of red jujube. Therefore, the fruit wine made from green jujube is not only nutritious, but also has a golden colour, a fruity aroma and a sweet taste [2]. At present, what is urgently needed to solve is the core production technology and rapid detection technology of fruit wine. This study focuses on the rapid detection of the continuous change information of alcohol content during fruit wine fermentation, so as to adjust the production process conditions in time, ensure the consistency and reproducibility of the fermentation process to the hilt, and ensure the high quality and stability of the final product.

Due to the time-consuming, complicated operation and low accuracy of traditional detection methods, it is difficult to meet the requirements of fast and timely production, while near infrared detection has the characteristics of fast detection speed, simple operation, high sensitivity and good reproducibility [3-5]. At present, the near infrared rapid detection technology has been explored and studied in the detection of alcohol. Zhang Shu Ming et al. used a combination of near infrared spectroscopy and electronic nose to analyse alcohol content in wine fermentation process [6], Jiao Ai Quan et al. used near infrared technology to quickly detect polyphenols and antioxidant capacity in yellow wine [7].

The current research on alcohol using near-infrared technology mainly focuses on the identification of wine, the detection of wine age, the analysis of total phenol, SSC and sugar [8-12]. Little work has been reported on the online rapid quantification of alcohol content in the fermentation process of fruit wine (especially jujube fruit wine). Therefore, the aim of this study was conducted to investigate the change of alcohol content during the fermentation of green jujube wine by near infrared technology, and to establish a rapid quantitative analysis mathematical model for the change of alcohol content in the fermentation process of jujube fruit wine. We expect

\footnotetext{
a Corresponding author: liguifeng99@163.com
} 
to provide a set of efficient, accurate and fast analytical methods for online quantitative detection of alcohol content during fruit wine fermentation.

\section{Materials and methods}

\subsection{Instruments}

Alcohol meter was produced in instrument factory of Wuqiang City in Hebei Province. NIR spectrophotometer (DA $7250^{\mathrm{TM}}$ ) was made in Perten company of Germany. All chemicals used were of analytical reagent grade with no further purification. Alcohol meter Hebei Province.

\subsection{Brewing process of jujube wine}

Completely jujube fruits were cleaned and then jujube pits were removed. The ratio of the green jujube pulp and water was $1: 2$, and softened in water bath at $90^{\circ} \mathrm{C}$ for 8-10 min. Jujube juice was collected by juice filtration then filtrated through gauze. Then, sugar was added to adjust the sugar content, and the mixture was inoculated with active dry yeast at $0.30 \mathrm{~g} / \mathrm{L}$. The fermentation temperature was controlled at $23-28^{\circ} \mathrm{C}$. The finished young wine was filtered through four layers of fine cheesecloth into $500 \mathrm{~mL}$ wine bottles that were subsequently sterilized. All samples were done in triplicate.

\subsection{Determination of alcohol content}

The alcohol content $(\%, \mathrm{v} / \mathrm{v}))$ was determined by alcohol meter (GB 5009. 225-2016) [13]. Each sample was repeated for 3 times, and the difference between the measurement results was within $0.5 \%$.

\subsection{NIR spectral information collection}

Jujube wine samples were filtered through the gauze and shaken well before the test in order to obtain homogenous samples prior spectroscopic analysis. For measurement of wine spectra, approximately $50 \mathrm{~mL}$ of each sample was investigated. DA $7250^{\mathrm{TM}}$ NIR spectrophotometer was used for the measurement of absorption of all wine samples in the NIR wavelength region from 950 to $1650 \mathrm{~nm}$ with a spectral resolution of $4 \mathrm{~cm}^{-1}$. The 175 average spectra obtained from single jujube wine samples, 100 were used for the calibration set, while 75 were used for the validation set. The background was measured at the beginning of each analysis using a reference substance (distilled water). Each sample was analysed in triplicate and the spectral average was considered for further analysis (Figure 1).

\subsection{Statistical analysis}

For statistical analysis, MATLAB version 8.0 and Microsoft Excel 2016 were used. The models of calibration were developed by using the data obtained from analytical determinations and the spectral data obtained from NIR spectra [14]. The 100 average spectra were used for the calibration set, while 75 were used for the validation set. The samples were selected at random. The partial least squares (PLS) regression models established by the training set, then was cross validated externally by using test set with MATLAB 8.0.

Several spectral pre-treatment algorithms were tested to remove light scattering interferences and other noise, including MSC (Multiplicative Scatter Correction), SRA (Stepwise Regression Analysis) and FD (First Derivative) treatment. PLS (Partial Least Squares) regression was applied to build quantitative models, using the spectral range 950-1650 nm. Models were then calibrated with the calibration set using the estimated number of latent variables. The independent set is then projected onto these models yielding the prediction set results. The PLS models' accuracy was evaluated using the root mean square error of calibration (RMSEC), the root means square error of cross validation (RMSECV) and the coefficient of determination of prediction $\left(\mathrm{R}^{2}\right)$. Several spectral pre-treatment methods were tested to process raw spectra aiming at removing unwanted spectral variations caused by baseline drifts, light scattering effects and temperature variations [15-17].

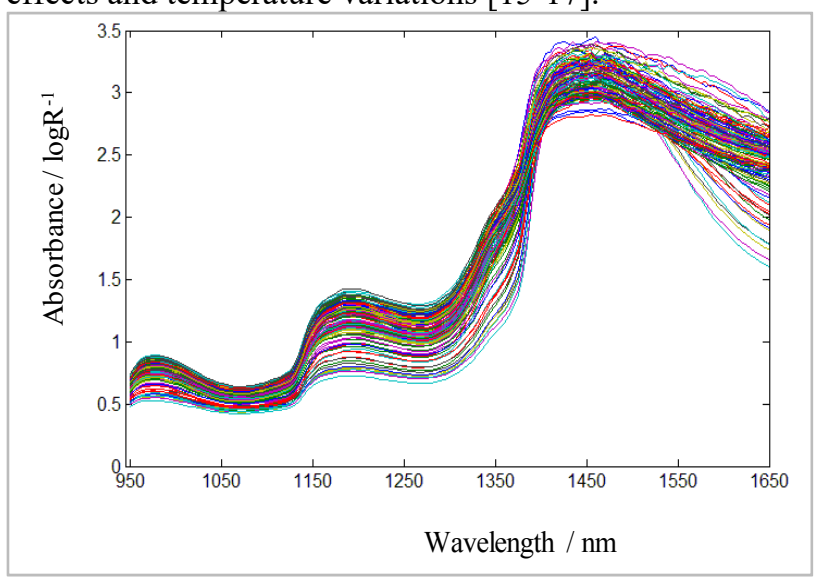

Figure 1. Original spectrogram

\section{Results and discussion}

\subsection{Spectral pre-treatment}

The spectral measurements were carried out on the basis of molecular overtone and the combination vibrations of stretch for the $\mathrm{CH}$ - and -OH groups, in the near infrared region. Therefore, the multivariate statistical method was employed to extract information from the recorded NIR spectra. The spectral pre-treatments, such as MSC (Figure 2) and FD (Figure 3) were applied to check the effect of spectral pre-treatments on the PLS regression model. For this optimization, the values of RMSEC, RMSECV and $\mathrm{R}^{2}$ were considered (Table 1 ). 


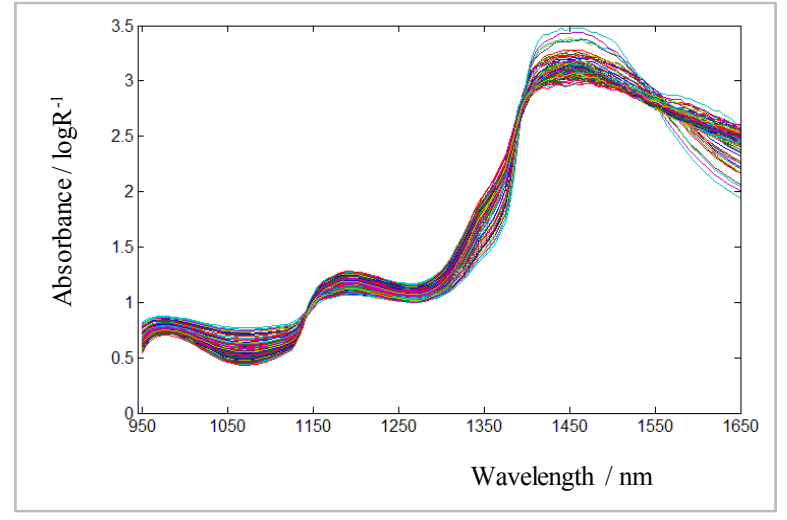

Figure 2. MSC pre-processing spectrogram

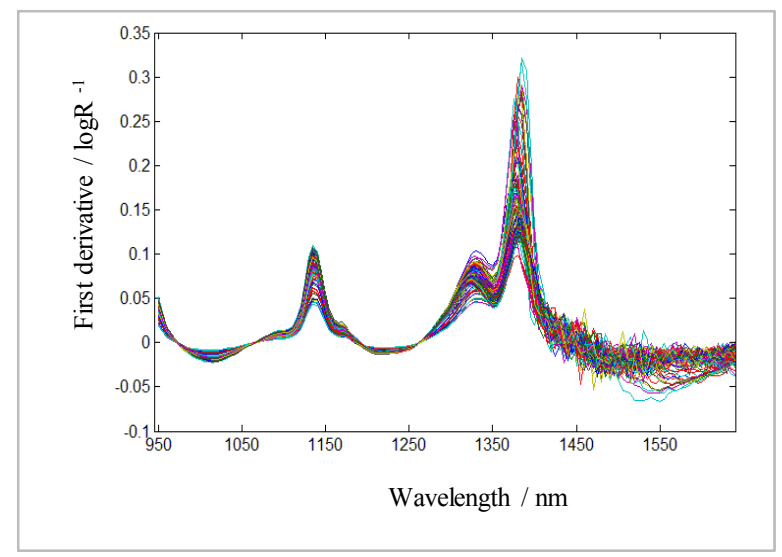

Figure 3. FD pre-processing spectrogram

Table 1. PLS calibration models for prediction of alcohol content of jujube wine using different spectral pre-treatments

\begin{tabular}{|c|c|c|c|}
\hline $\begin{array}{c}\text { Pre-treatment } \\
\text { methods }\end{array}$ & RMSEC & RMSECV & $\mathrm{R}^{2}$ \\
\hline $\begin{array}{c}\text { Original } \\
\text { spectrum }\end{array}$ & 0.7125 & 0.7742 & 0.8246 \\
\hline FD & 0.6763 & 0.6970 & 0.9083 \\
\hline MSC & 0.6827 & 0.7253 & 0.8937 \\
\hline
\end{tabular}

\subsection{Select the optimum wavelength}

The jujube wine samples' NIR spectra were divided into four regions: region $1(950-1050 \mathrm{~nm})$, region $2(1050$ $1250 \mathrm{~nm})$, region $3(1250-1300 \mathrm{~nm})$ and region $4(1300-$ $1650 \mathrm{~nm})$. This division was set to understand the regions with major influence for estimating the alcohol content. the best wavelengths were chosen using the stepwise algorithm in MATLAB (Table 2).

Table 2. The optimal wavelength table by stepwise regression

\begin{tabular}{|c|c|}
\hline $\begin{array}{c}\text { Pre-treatment } \\
\text { methods }\end{array}$ & The optimum wavelengths \\
\hline
\end{tabular}

\begin{tabular}{|c|c|}
\hline MSC & $\begin{array}{c}96597510801105112011801200 \\
153015551650\end{array}$ \\
\hline \multirow{2}{*}{ FD } & $\begin{array}{c}9651060117011901390 \\
14001465148015601605\end{array}$ \\
\hline
\end{tabular}

\subsection{Establishment of model}

PLS regression model, which is considered a classic multiple linear regression model, was applied successfully as a tool of calibration, validation, and prediction in the form of a multivariate method. The stepwise regression analysis was applied to investigate the relationship between alcohol content and wavelength. The PLS regression model was built by using the calibration set. The PLS models' results (Figure 4 and Figure 5) showed an excellent agreement between NIR spectra predictions and all chemical procedures. The best PLS models were obtained for alcohol content $\left(\mathrm{RMSEC}=0.6572, \mathrm{RMSECV}=0.6970\right.$ and $\left.\mathrm{R}^{2}=0.9246\right)$.

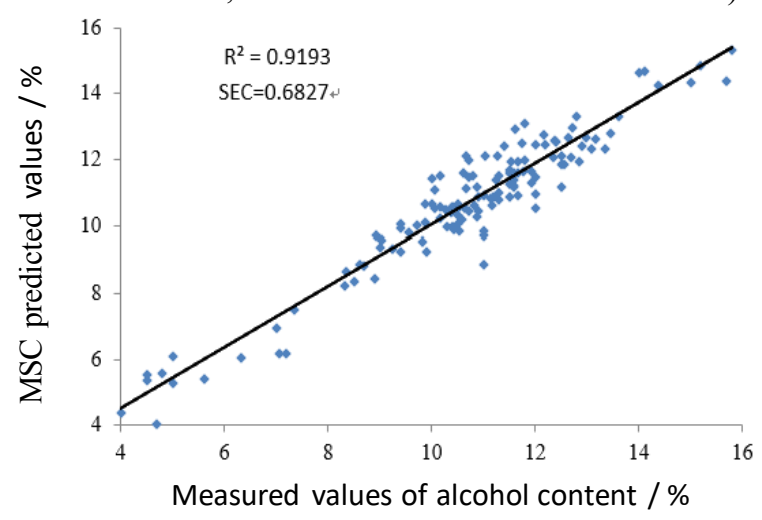

Figure 4. Correlation between predicted values of the MSC pre-processing models and actual values

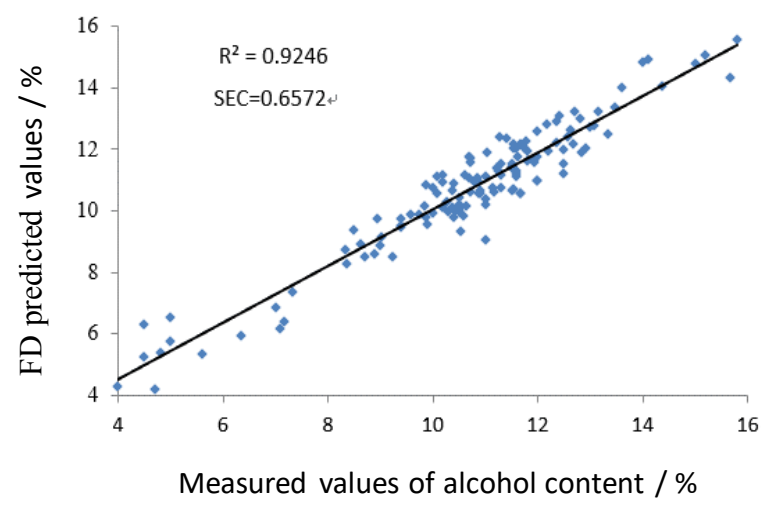

Figure 5. Correlation between predicted values of the FD preprocessing models and actual values

\subsection{Validation of model}

In order to confirm the validity and reliability of the optimal model, the samples of external validation set (50 samples) was determined. The prediction capacity of the model obtained was assessed by with the ratio performance deviation, based on the comparison between the NIRS model predicted values and the 
measured values by alcoholometer (Figure 6). Data difference of predicted values and the measured values was less than $1.3 \%$. This means that the analysis results were satisfactory. These results suggested that the NIR calibration was a very interesting tool for rapid analysis of alcohol content during the green jujube wine fermentation.

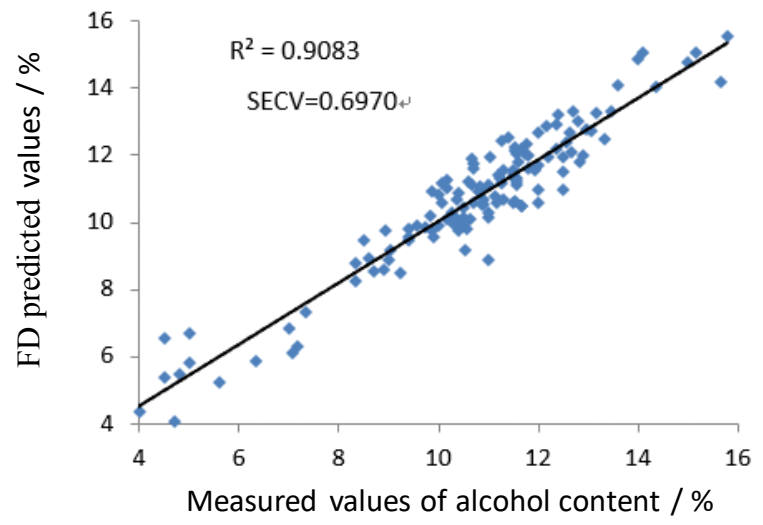

Figure 6. Correlation between predicted values of the FD optimal model and actual values (external validation set)

\section{Conclusion}

In this work, NIR spectroscopy was used to determine the alcohol contents of jujube wine samples $(n=175)$. The multivariable analyses including FD-PLS and MSCPLS were conducted to build the regression models. The excellent model was with high coefficient of determination $\left(\mathrm{R}^{2}=0.9246\right)$ and low $\operatorname{RMSEC}(0.6572)$. The results obtained in this work showed that NIR spectroscopy was able to predict the alcohol contents of jujube wine in a fast, reliable and accurate way.

\section{Funding}

This work was financially supported by project of the Food nutrition and health quality program of Shanxi Normal University (No. 2018YZKC-07) and National innovation training program for College Students (No. 201910118014).

\section{Acknowledgments}

Authors are whishing thankful to the teachers and students of research group.

\section{References}

1. X. F. Zhou, X. F. Guo, Y. F. Feng. Z. Q. Wang, C. Y. Wu. Science and Technology of Food Industry, 39, 296-300. (2018)

2. D. Cheng, C. Q. Zhu, J. K. Cao, W. B. Jiang. Food and Chemical Toxicology, 50, 1302-1308. (2012)

3. B. Y. Kwan, M. S. Kim, I. S. Baek, D. Y. Kim, W. H. Lee, J. k. Kim, H. H. Bae, Y. S. Kim. Postharvest Biology and Technology, 76, 40-49. (2013)
4. I. Esteban-Diez, J. M. Gonzalez-Saiz, C. Pizarro. Journal of near Infrared Spectroscopy, 12, 287-297. (2004)

5. A. D. Girolamo, C. V. Holst, M. Cortese, S. Cervellieri, M. Pascale, F. Longobardi, L. Catucci, A. C. Raffaella-Porricelli, V. Lippolis. Food Chemistry, 282, 95-100. (2019)

6. S. M. Zhang, Y. Yang, Y. Y. Ni. Spectroscopy and Spectral Analysis, 32, 2997-3001. (2012)

7. A. Q. Jiao, X. M. Xu, Z. Y. Jin. Journal of Food Science and Biotechnology, 35, 357-363. (2016)

8. F. Shen, Y. B. Ying, B. B. Li. Food Science, 35, 2528. (2014)

9. Y. M. Wang, J. L. Zhu, H. Zhang, J. X. Zhao, X. H. $\mathrm{Gu}, \mathrm{H}$. X. Zhu. Chinese Journal of Luminescence, 39, 1310-1316. (2018)

10. D. f. Jie, L. j. Xie, X. P. Fu, X. Q. Rao, Y. B. Ying. Journal of Food Engineering, 118, 387-392. (2013)

11. M. Urbano-Cuadrado, M. D. Luquede-Castro, P. M. Pérez Juan, M. A. Gómez-Nieto. Talanta, 66, 218224. (2005)

12. D. Cozzolino, M. J. Kwiatkowski, M. Parker, W. U. Cynkar, R. G. Dambergs, M. Gishen, M. J. Herderich. Analytica Chimica Acta, 513, 73-80. (2004)

13. GB 5009. 225-2016, China Standards Press. (2016)

14. G. F. Li, G. J. Zhao, X. D. Wang, X. H. Liu. Transactions of the Chinese Society of Agricultural Engineering, 6, 169-173. (2008)

15. C. Li, T. Zhao, C. Li, L. Mei, E. Yu, Y. Dong, J. Chen, S. Zhu. Food Chemistry, 221, 990-996. (2017)

16. G. F. Li, G. J. Zhao, X. H. Liu, C. L. Xiao. Transactions of the Chinese Society for Agricultural Machinery, 40, 120-123. (2009)

17. L. Li, X. Y. Dong, H. Wu, C. Zhang, Y. Zhou, M. B. Chen, S. L. Fang, Y. Q. Xie, Y. Y. Jing, Y. Zhang. Liquor Making, 45, 57-60. (2018) 